\title{
Biographies et histoires de patients
}

\section{Eberhard Wolff}

PD Dr rer. soc., rédacteur culture, histoire, société

Comment raconteriez-vous l'histoire de votre vie? Selon vous, votre parcours était-il prédestiné ou est-il le résultat du hasard, est-ce vous qui l'avez configuré ou les autres? Parleriez-vous de l'histoire d'un succès, d'un doute, d'une découverte de

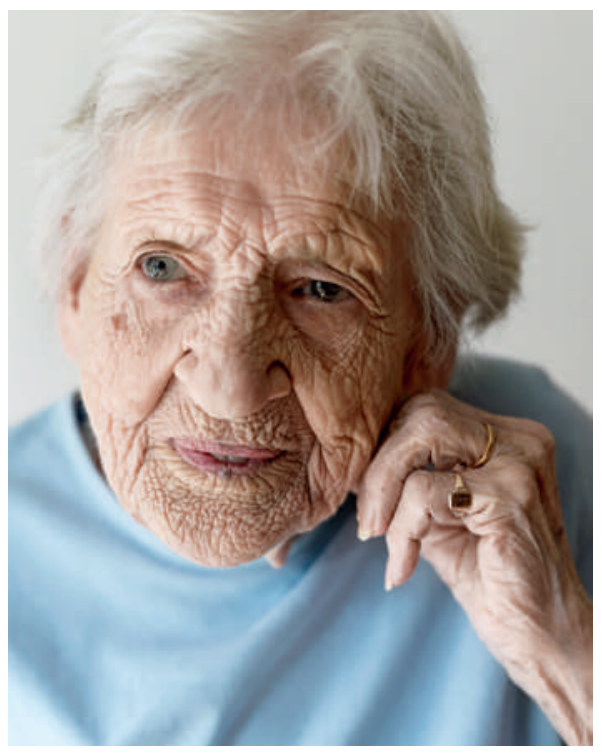

Claire Parkes-Bärfuss - photographiée par Mara Truog qui a reçue pour cette image le Swiss Press Award 2016 dans la catégorie «Portrait». vous-même, ou d'une affirmation de vous-même?

Les maladies marquent particulièrement les itinéraires. Mais c'est aussi un matériel dont l'interprétation est nécessaire pour raconter une biographie. Prenons un exemple. Claire Parkes-Bärfuss a eu une vie impressionnante. Née en 1913, dans un milieu modeste du Laufonnais, elle passe sa jeunesse en foyer puis travaille comme employée de maison et ne parvient jamais à vraiment concrétiser son aspiration professionnelle, à savoir devenir «infirmière». A 36 ans, elle quitte la Suisse pour l'Angleterre, avant de partir en Nouvelle-Zélande, puis de revenir en Angleterre. Plus d'un demi-siècle plus tard, elle est de retour en Suisse, à 99 ans! C'est là qu'elle a raconté sa vie à la journaliste Simone Müller. Cette dernière en a tiré une sorte d'autobiographie de second degré, dans un livre qui fut un succès [1]. Une biographie sensible et captivante, méritant d'être lue, sur la pauvreté et le travail, encore et toujours le travail, sur l'incessante adaptation à un environnement en permanente mutation, sur la quête incessante d'une vie stable, et sur une courageuse affirmation de soi dans un contexte difficile.

La maladie couvre une part importante de cette biographie. Dans les plus jeunes années de sa vie, la tuberculose est le «fil rouge» de l'existence de la jeune Klara Bärfuss: la phtisie de beaucoup de ses proches; la tuberculose de sa mère, veuve, qui entraîna le départ des frères et sœurs et leur placement dans un foyer très strict; enfin le décès de sa mère et de sa grande sœur, à cause de la tuberculose. La maladie est ici racontée comme un singulier coup du sort.

Ensuite, une faiblesse pulmonaire, attestée par un médecin mais inventée, a sauvé Klara d'une famille affreuse où elle travaillait. Cela amène Klara au service d'une autre maison, vers Davos. La maladie est alors racontée comme une chance dans la vie.

En Angleterre, Klara, entretemps devenue «Claire», s'occupe pendant un moment des malades atteints de tuberculose, comme aide-soignante au London Chest Hospital. La maladie est ici racontée comme un outil pour forger ses propres expériences et réaliser ses vœux. Puis suit son mariage avec l'Anglais Stanley Parkes et l'émigration vers la Nouvelle-Zélande, avec une petite aisance. La vie de couple est difficile. L'époux, intérieurement fragile, coupe une nouvelle fois avec les racines qu'ils avaient plantées en devenant propriétaire de leur petite maison. Ils retournent en Angleterre. Et la maladie redevient le fil rouge expliquant les fractures biographiques. Les anciennes expériences traumatisantes du mari pendant la guerre entretiennent l'instabilité du couple et de l'existence de ces deux êtres. La maladie est maintenant racontée comme un frein au bonheur.

A plus de soixante-dix ans, alors que, devenue veuve, Claire habite une résidence anglaise pour personnes âgées, sa vue s'amenuise. Elle devient pratiquement aveugle. Cela ne l'empêche pas de revenir en Suisse deux décennies plus tard, puis de retourner en Angleterre pour finalement s'installer définitivement en Suisse. Même malvoyante, elle fait la lessive et cuisine elle-même ses repas, à presque cent ans. La perte de la vue sert de fil rouge à la vieillesse. Mais pas comme un calvaire, plutôt comme un symbole d'énergie vitale de cette femme qui n'a «jamais abandonné» et "continue toujours à avancer». La maladie est ici racontée comme l'histoire d'une affirmation de soi.

L'histoire des personnes n'est pas spontanée, chacun se la forge soi-même: c'est le message du titre d'un ouvrage de 1984 («Die Menschen machen ihre Geschichte nicht aus freien Stücken, aber sie machen sie selbst»), et aussi celui de l'historien allemand Lutz Niethammer (dans une critique qui fait un clin d'œil à Karl Marx). C'est la même chose pour les histoires de maladies. Elles ne se contentent pas d'exister. Elles sont aussi forgées par notre action. Plus encore, dans le récit, elles sont vraiment créées comme de simples histoires. Pas à titre exceptionnel, mais de façon récurrente. Et toujours nouvelle, quelle que soit la personne qui les écrit. Même dans les futurs dossiers électroniques des patients. 